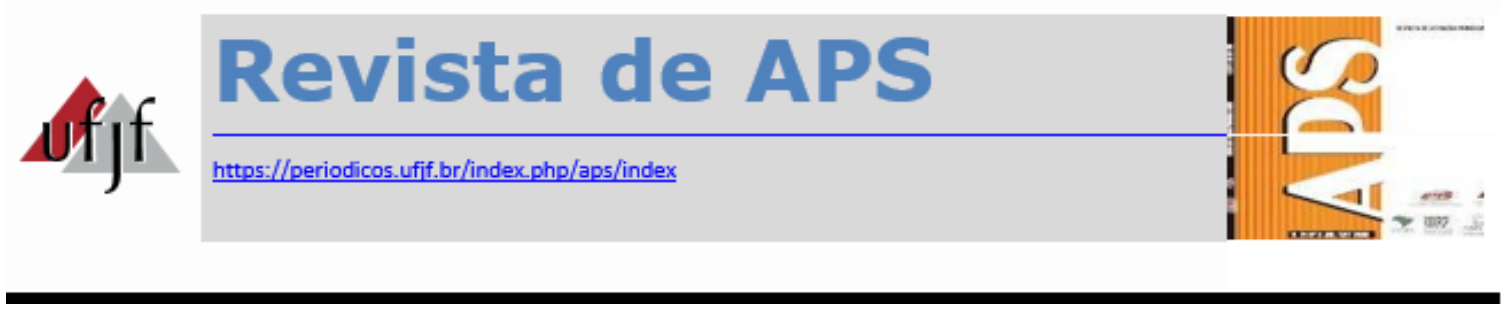

\title{
Percepção das beneficiárias sobre o Programa Brasileiro de Prevenção e Controle da Deficiência de Vitamina A
}

\section{Beneficiaries' perception regarding the Brazilian Program of Prevention and Control of Vitamin A Deficiency}

Dixis Figueroa Pedraza ${ }^{1}$, Juliane Berenguer de Souza Peixoto², Maria Gorete Lucena de Vasconcelos ${ }^{3}$, Virgínia Rossana de Sousa Brito ${ }^{4}$, Adriana de Azevedo Paiva ${ }^{5}$

\begin{abstract}
RESUMO
Objetivo: compreender a percepção das beneficiárias sobre o Programa Nacional de Suplementação de Vitamina A no município de Campina Grande, no estado da Paraíba, no Brasil. Métodos: estudo qualitativo com amostra de 25 sujeitos definida pelo processo de saturação teórica. A coleta ocorreu por meio de entrevistas, utilizando-se roteiro com questões norteadoras sobre o Programa, que foram gravadas em aparelho de áudio. Após transcrição na íntegra, as falas foram submetidas a análise de conteúdo. Resultados: a percepção das beneficiárias mostrou saber popular sobre a vitamina $A$, mecanicidade da suplementação e desconhecimento e invisibilidade do Programa. Conclusão: as falas revelaram percepção das beneficiárias de desconhecimento e invisibilidade do Programa e de seus componentes.
\end{abstract}

PALAVRAS-CHAVE: Atenção Primária à Saúde. Estratégia Saúde da Família. Deficiência de Vitamina A. Percepção. Programas e Políticas de Nutrição e Alimentação.

\footnotetext{
${ }^{1}$ Docente do Curso de Enfermagem da Universidade Estadual da Paraíba. Professor doutor. E-mail: dixisfigueroa@gmail.com.

${ }^{2}$ Docente do Curso de Enfermagem da Faculdade União de Ensino Superior de Campina Grande (PB). Professora mestre.

${ }^{3}$ Docente do Programa de Pós-graduação em Saúde da Criança e do Adolescente da Universidade Federal de Pernambuco. Professora doutora.

${ }^{4}$ Docente do Curso de Enfermagem da Universidade Estadual da Paraíba. Professora doutora.

${ }^{5}$ Docente do Curso de Nutrição da Universidade Federal do Piauí. Professora doutora.
} 


\begin{abstract}
Objective: understanding the perception of the beneficiaries regarding Vitamin A Supply National Program in the municipality of Campina Grande, Paraíba, Brazil. Methods: a qualitative study with a sample of 25 subjects defined by a theoretical saturation process. The data collection was carried out by means of interviews, using a script comprising of guiding questions about the Program, which were recorded on an audio device. After fully transcribed, the speeches were submitted to content analyses. Results: the perception of the beneficiaries showed lay knowledge about vitamin A, mechanicity of the supplementation and unfamiliarity and invisibility of the Program. Conclusion: the interviews revealed that according to the perception of the beneficiaries the Program and its components were unfamiliar and invisible to them.
\end{abstract}

KEYWORDS: Primary Health Care; Family Health Strategy; Vitamin A Deficiency; Perception; Nutrition Programs and Policies.

\title{
INTRODUÇÃO
}

A vitamina A é um micronutriente importante para o funcionamento adequado do sistema visual, a manutenção da função e da integridade celular, o crescimento corporal adequado, a eritropoese, a imunidade e a reprodução. ${ }^{1,2}$ Sua carência pode causar manifestações clínicas e/ou subclínicas, genericamente denominadas deficiência de vitamina A (DVA), e contribui significativamente para a morbimortalidade maternoinfantil e a gravidade das infecções comuns da infância, inclusive em nível subclínico. ${ }^{3}$

As principais causas da DVA são a alimentação inadequada e os processos infecciosos. A alimentação inadequada pode proporcionar déficit na ingestão de alimentos fontes de vitamina $A$ e o consumo insuficiente de alimentos contendo nutrientes importantes para seu bioaproveitamento, o que pode ser influenciado por fatores culturais, condições socioeconômicas e preferências alimentares. ${ }^{2}$ Consideramse as gestantes, as lactantes e as crianças em idade pré-escolar os grupos de maior vulnerabilidade à DVA, pela maior necessidade requerida do micronutriente nesses períodos. ${ }^{4}$

No Brasil, a DVA é considerada um problema de saúde pública que não está restrito a áreas geográficas economicamente desfavorecidas, ${ }^{3}$ afetando crianças e mulheres em idade fértil de todas as regiões brasileiras. ${ }^{4,5}$ Os dados da Pesquisa Nacional de Demografia e Saúde (PNDS) de 2006 mostraram que 59,5\% das crianças brasileiras menores de cinco anos apresentavam níveis baixos de retinol sérico, concentrando as maiores taxas no Nordeste $(19,0 \%)$ e no Sudeste $(21,6 \%)$ do país. ${ }^{5}$ No 
estado da Paraíba, uma pesquisa estadual em nove municípios, entre eles Campina Grande, observou prevalência de 21,8\% de DVA em crianças de 6 a 59 meses de idade da zona urbana. ${ }^{2}$ Outros resultados mais recentes atestam a manutenção da carência nutricional, ${ }^{6,7}$ e análises complementares da PNDS indicaram associação da DVA nas crianças a residência em zona urbana e ter mãe com idade de 36 anos ou mais. ${ }^{8}$

Os países afetados pela DVA têm adotado estratégias de prevenção, monitoramento e tratamento, focalizando, principalmente, os grupos mais vulneráveis. Tais estratégias consistem em suplementação de megadoses de vitamina $A$, fortificação de alimentos com vitamina A e incentivo a diversificação alimentar, todas elas vinculadas à educação alimentar e nutricional. ${ }^{3,9}$

O Brasil iniciou, a partir de 1983, intervenções de combate e prevenção da DVA. A estratégia utilizada na época foi a administração de megadoses de vitamina A para pré-escolares nos dias nacionais de vacinação, em áreas específicas consideradas de alto risco para DVA. ${ }^{1,10}$ Posteriormente, o Programa Nacional de Suplementação de Vitamina A (PNVITA) foi instituído em 2005 com o objetivo de reduzir e controlar a DVA entre crianças de 6 a 59 meses de idade e puérperas no pós-parto imediato. Incialmente restrito a municípios do Nordeste, em 2010 o programa foi estendido para os municípios que compõem a Amazônia Legal. Em 2012, o governo federal, por meio da ação Brasil Carinhoso, expandiu o PNVITA para todos os municípios da região Norte, 585 municípios integrantes do Plano Brasil sem Miséria das regiões Centro-Oeste, Sul e Sudeste e todos os Distritos Sanitários Especiais Indígenas, ampliando, assim, a ação de combate à DVA. ${ }^{4}$

Estudos sobre o impacto do PNVITA que analisaram o grau de conhecimento das famílias beneficiadas em relação à vitamina $A$ e ao funcionamento do Programa detectaram um grande desconhecimento sobre ele, a vitamina A e a DVA, bem como falhas nas práticas de educação em saúde, fatos que, segundo os autores, podem interferir na adesão da população-alvo às ações do Programa, na operacionalização deste e em seus resultados em termos de cobertura e prevalências da DVA. ${ }^{10,11}$ Outra pesquisa realizada no estado da Paraíba mostrou falhas em relação à operacionalização do Programa em nove cidades do estado. Tal fato evidenciou, sobretudo, a necessidade de fortalecimento das ações educativas junto aos profissionais da saúde e à populaçãoalvo. ${ }^{12}$ Entretanto, apesar da importância desses resultados, ainda são poucos os estudos que objetivaram avaliar o Programa na tentativa de explicar a persistência das 
altas taxas de DVA. ${ }^{11,12}$ Desse modo, este estudo teve por objetivo compreender a percepção sobre o Programa entre beneficiárias gestantes e responsáveis por crianças de 6 a 59 meses no município de Campina Grande, no estado da Paraíba, no Brasil.

\section{CASUÍSTICA E MÉTODOS}

O presente estudo é um recorte da pesquisa "Intervenção educativa envolvendo os profissionais da saúde e a população-alvo: impacto na operacionalização e nos resultados do Programa Nacional de Suplementação de Vitamina A no município de Campina Grande-PB", cujo objetivo foi avaliar o impacto de uma intervenção de capacitação de profissionais da saúde e de educação alimentar e nutricional da população-alvo na operacionalização e nos resultados do PNVITA. A pesquisa partiu de uma avaliação multidimensional que compreendeu as dimensões da estrutura, do processo e dos resultados do Programa. Tal diagnóstico incluiu a análise da percepção dos usuários sobre o Programa, o qual é abordado neste trabalho. Trata-se de um estudo de natureza analítica e interpretativa de informações qualitativas, realizado no cenário das Unidades Básicas de Saúde da Família (UBSF) no município de Campina Grande (PB).

A cidade de Campina Grande está localizada na região do agreste da Paraíba, distante 120 km de João Pessoa, capital do Estado. A população estimada do município era de 385.276 habitantes em 2010. ${ }^{13}$ No mesmo ano, a cidade dispunha de 78 Centros de Saúde/Unidades Básicas de Saúde, perfazendo um total de 91 Equipes de Saúde da Família que cobriam, em média, $83 \%$ da população do município, segundo os dados do Cadastro Nacional de Estabelecimentos de Saúde. ${ }^{14}$

Foram considerados elegíveis para o estudo dois grupos: gestantes e responsáveis por crianças de 6 a 59 meses, com idades superiores a 18 anos. A opção pelas gestantes, no lugar das puérperas, ocorreu como forma de viabilizar a coleta de dados, bem como de implementar a intervenção, proposta da pesquisa, antes que elas recebessem a suplementação com vitamina $A$, que é administrada no pós-parto imediato ou no momento da alta hospitalar. ${ }^{4}$ Os sujeitos do atual estudo foram nove gestantes e 16 responsáveis por crianças de 6 a 59 meses de idade, o correspondente ao processo de saturação teórica, ${ }^{15}$ atendidas em oito UBSF do município que 
participaram da intervenção. Elas foram selecionadas por sorteio simples e alocadas randomicamente em grupo de intervenção e grupo-controle.

Coletaram-se os dados no período de novembro de 2009 a junho de 2010, por meio de entrevistas individuais ocorridas em salas fechadas das UBSF. Optou-se pelas entrevistas, em virtude de elas possibilitarem o aprofundamento dos aspectos de interesse por meio da abordagem de pessoas dos mais diversos graus de escolaridade, já que não exigem leitura nem escrita do entrevistado, pois as perguntas e as respostas são realizadas oralmente. ${ }^{16}$

As usuárias dos serviços de saúde tiveram, inicialmente, dados coletados em relação ao perfil sociodemográfico, que compreendeu informações sobre escolaridade, situação conjugal, exercício de atividade laboral, número de moradores na residência, número de moradores na residência que trabalham, renda familiar, tipo de moradia, tipo de construção da residência e existência de saneamento básico na residência e tipo de coleta de lixo. Utilizou-se um roteiro de entrevista com seis questões norteadoras sobre o PNVITA: I - O que é a vitamina A; II - O que é a deficiência de vitamina A?;III Fale sobre o que conhece do programa da vitamina A; IV - . Você já viu algum cartaz, figura ou ilustração com orientações sobre a vitamina $A$ ou o programa da vitamina $A$ ?; V - Se você participa ou participou de atividades educativas sobre alimentação e nutrição no posto de saúde, fale sobre elas; e VI - Você acha que o programa da vitamina A é importante? As entrevistas tiveram tempo médio de duração de 30 minutos e foram gravadas em aparelho de áudio.

As falas foram transcritas na íntegra e submetidas à análise de conteúdo, modalidade temática, de acordo com os seguintes passos: I - Leitura exaustiva das falas com o intuito de apreender os núcleos de sentido; II - Agrupamento das ideias centrais; III - Construção e interpretação das categorias relacionadas à concepção expressa nas ideias. $^{17}$

Atendendo à Resolução no 196/96 do Conselho Nacional de Pesquisa, solicitouse a anuência institucional para a realização da pesquisa e encaminhou-se para a apreciação e o parecer do Comitê de Ética em Pesquisa da Universidade Estadual da Paraíba, processo aprovado sob protocolo no 0050.0.133.000-08. Os sujeitos do estudo assinaram o Termo de Consentimento Livre e Esclarecido, em duas vias, uma das quais ficou sob suas posses. Para garantir o anonimato, as entrevistadas foram identificadas 
em suas falas pela letra $E$, seguida do número correspondente à ordem cronológica da entrevista.

\section{RESULTADOS}

A amostra foi constituída $100 \%$ por mulheres. De acordo com os dados socioeconômicos e demográficos das entrevistadas, pode-se observar um perfil majoritário de mulheres em vulnerabilidade. Das 25 participantes do estudo, apenas cinco haviam concluído o ensino médio, sendo a principal ocupação os afazeres do lar $(n=20)$. Entre as que desenvolviam atividade laboral, as profissões relatadas foram as de babá e lavadeira. A maioria vivia com companheiro $(n=17)$, e o número de pessoas residentes no domicílio variou de duas a 10 pessoas.

Na maioria dos domicílios $(n=14)$, apenas uma pessoa contribuía com a renda familiar. A renda mensal per capita menor que $1 / 4$ de um salário mínimo $(n=17)$ foi predominante entre as entrevistadas. Quanto à moradia, a própria e a alugada foram predominantes $(n=8)$, enquanto seis das famílias moravam em casa emprestada. A maioria dos domicílios possuía água encanada proveniente da rede pública, salvo uma entrevistada que relatou não ter. Do total, 15 tinham esgotamento sanitário da rede pública, oito possuíam fossa e duas não recebiam tratamento de seus dejetos, ficando a céu aberto. As falas e o agrupamento de ideias, analisados de acordo com o objetivo do estudo, levaram à formação de três categorias que representam o contexto da percepção das gestantes e responsáveis por criança de 6 a 59 meses do PNVITA.

\section{Categoria I - Conhecimento popular}

O conhecimento da população-alvo sobre a vitamina A é essencial para o reconhecimento da sua importância. As falas das entrevistadas expressaram sentidos do conhecimento popular com foco nas fontes alimentares e funções do micronutriente:

[...] Leite Ninho tem vitamina A e outras coisas também [...]. Vem explicando na embalagem (E1).

[...] Frutas, verduras, legumes, têm várias que têm vitamina $A$ (E5).

[...] A vitamina A é importante para a queda de cabelo, né? Pra prevenir, né? (E12).

[...] Já ouvi falar. [...] Tem que comer muita fruta e verdura pra ficar 
forte, pra ficar bem. [...] Eu que sei, escuto falar na minha casa também, minha mãe comentava (E15).

[...] Vitamina A é boa para amamentar. [...] É só pra ajudar no leite (E10).

\section{Categoria II - Mecanicidade da suplementação}

As falas das entrevistadas expressaram a mecanicidade do ato da administração do suplemento de vitamina A pelos profissionais de saúde. Assim, desenvolve-se uma atividade longe da abordagem da promoção da saúde e do autocuidado:

[...] Não diz nada (referindo-se ao profissional); só faz abrir a boca dela e pronto. E marcar no cartão (E3).

[...] Eu só dava vitamina, né? [...] Eu não tinha explicação [...] quando tinha campanha. Eu vinha e dava (E5).

[...] Eles (os profissionais) deram vacina de vitamina A, mas nunca me falaram, não. Ninguém nunca me explicou (E8).

[...] Sei que ele deve tomar a vitamina A quando a Agente de Saúde vai lá em casa e diz o dia que ele tem que tomar (E2).

\section{Categoria III - Sinalização do usuário sobre limitações do Programa}

O desconhecimento do Programa e a possibilidade de administração irregular da vitamina A foram revelados:

[...] Sobre o Programa da vitamina A , não sei nada, não (E1).

[...] Não, nunca ouvi (referindo-se ao Programa). É isso que eu te disse, eu só fazia vacina no menino, mas ninguém nunca me disse, ninguém nunca falou (E5).

[...] Conheço, não. Vem tomar e pronto, toma (E12).

Nesta categoria, também foi possível distinguir discrepâncias de opiniões em relação à regularidade na administração da vitamina $A$ :

[...] Já aconteceu uma vez de faltar a vitamina A (E7).

[...] Já faltou. Só uma vez, só (E12).

[...] Nunca faltou. Sempre teve a vitamina (E2).

[...] Sempre tem. [...] A gente sempre faz o acompanhamento quando tem vacina $(E 3)$.

\section{DISCUSSÃO}

A percepção de gestantes e responsáveis por crianças de 6 a 59 meses sobre o PNVITA no município do estudo mostrou conhecimento elementar sobre a vitamina A e 
desconhecimento do Programa enquanto conjunto de ações e estratégias para a prevenção e o controle da DVA. No que diz respeito ao conhecimento sobre a vitamina A, percebeu-se o predomínio de saberes populares ou de senso comum que devem ser acolhidos pelos profissionais de saúde como complemento do conhecimento científico e no planejamento das suas ações. ${ }^{18,19}$ Entretanto, de acordo com uma pesquisa sobre o tema, apenas uma minoria dos profissionais de saúde valoriza e utiliza os conhecimentos populares como complementares aos conhecimentos científicos, o que é importante na aceitação do serviço. ${ }^{18}$

Trabalhar na perspectiva da educação popular possibilita um diálogo com o usuário, respeitando-se e valorizando-se seus saberes, rompendo-se com o conhecimento verticalizado e contemplando-se a troca de informações e experiências. Permite, além de uma ligação entre o conhecimento científico e o popular, favorecer o autocuidado, a prevenção de doenças e a promoção da saúde individual e coletiva. ${ }^{20}$

O profissional de saúde inserido no PNVITA, trabalhando a partir do conhecimento popular, pode valorizar e resgatar a cultura alimentar da comunidade sob a perspectiva da educação alimentar e nutricional e da promoção da saúde. ${ }^{21}$ Assim, no contexto deste trabalho, é importante ressaltar que os profissionais de saúde devem considerar a valorização que os usuários fizeram de alimentos de alto valor nutricional no sentido de destacar a importância da diversificação alimentar, com ênfase no consumo de alimentos fontes de vitamina A. Esse consumo pode ser garantido com o fomento à produção e ao cultivo desses alimentos, levando em consideração a diversidade regional brasileira e considerando estratégias como as hortas caseiras, comunitárias ou escolares. Dessa forma, a promoção e a prevenção da DVA podem ser construídas a partir das concepções culturais da comunidade atendida com mudanças no comportamento da população.

A categoria temática mecanicidade da suplementação da vitamina A revelou uma prática realizada pelos profissionais de saúde do município longe da abordagem da promoção da saúde e do autocuidado que enfraquece o PNVITA. É preciso que a suplementação seja acompanhada de orientações com atividades educativas voltadas para a promoção da saúde como ferramenta importante para garantir a participação popular na corresponsabilização pela adoção de hábitos alimentares saudáveis, com reflexos na comunidade, na família, e não só no indivíduo. ${ }^{22}$ 
Similarmente, uma avaliação do PNVITA realizada no estado da Bahia detectou que as atividades educativas não aconteceram e/ou não produziram os resultados esperados. ${ }^{10}$ Tal estudo deixou clara a necessidade da criação de novas estratégias para o desenvolvimento da educação nutricional de forma permanente e por pessoal treinado no Programa de Combate à DVA, tal como concebido por outros autores. ${ }^{21}$ Os relatos encontrados na Bahia reforçam a ideia de que a estratégia adotada pelo Programa de associar a distribuição de megadoses de vitamina A à imunização não favorece o esclarecimento à população sobre a importância deste micronutriente na manutenção da saúde dos grupos vulneráveis. ${ }^{10}$ Ainda, os resultados convergem com o relato histórico das ações educativas nos programas oficiais de combate à DVA no Brasil, nos últimos quarenta anos, que mostrou a não realização ou a realização temporária e sem avaliação. ${ }^{21}$

Resultados derivados desta mesma pesquisa, segundo os profissionais, revelaram, efetivamente, que a suplementação das megadoses da vitamina $A$ é percebida como uma ação isolada, e não como parte de um conjunto de atividades preconizadas pelo Programa. ${ }^{23}$ A capacitação dos profissionais sobre o PNVITA é essencial à sua operacionalização. É importante que os profissionais conheçam e usem o manual de condutas do Programa, no qual se estabelece a importância de informar, comunicar e educar a população-alvo, ${ }^{4}$ de tremenda importância na população observada que manifestou claramente a falta de acesso à informação.

Em relação à categoria temática referente à sinalização do usuário sobre limitações do Programa, ficou latente o desconhecimento do Programa. A não identificação da existência do Programa pode comprometer sua sustentabilidade, uma vez que este não é reconhecido como direito e ação de saúde. ${ }^{11}$

De maneira semelhante, outros estudos ${ }^{10-12}$ mostraram pouca visibilidade do PNVITA para a população-alvo. Sob tal contexto, é importante ressaltar que ações educativas relacionadas ao Programa devem orientar e sensibilizar os responsáveis quanto à importância da vitamina $\mathrm{A}$, de sua suplementação e da diversificação alimentar para a saúde da criança. ${ }^{11}$ Nesse sentido, estudiosos do tema consideram o serviço de puericultura das unidades de saúde o momento mais apropriado para desenvolver o processo de comunicação social destinado à popularização da importância da vitamina A e das consequências de sua deficiência na população-alvo. ${ }^{10}$. 
Ainda com relação à categoria temática, vale enfatizar que as opiniões opostas em relação à regularidade da suplementação podem ter justificativas na possibilidade de a escassez da vitamina $A$ nas unidades de saúde não ser percebida pela populaçãoalvo por falta de conhecimento sobre o Programa, sobre a periodicidade na aplicação do suplemento e sobre os grupos beneficiários. Tais fatos podem ser comprovados nas seguintes falas:

[...] A criança deve tomar a vitamina A sempre na campanha. Eu só dei assim (E1).

[...] Acho que é de mês em mês... Ou é de ano em ano... (E11).

[...] Acho que todos. Todas as crianças, o idoso (...). Por que é mais frágil, né, pega doença (E16).

[...] Ele (referindo-se a seu filho) tomou esse mês ainda. Foi vacina, injeção (E17).

A suposição pode, ainda, ser sustentada segundo a percepção dos profissionais que revelaram a falta da vitamina A como uma das limitações que dificultam a operacionalização do Programa no município de Campina Grande. ${ }^{23}$ Nesse contexto, o estudo realizado no estado da Paraíba revelou cobertura insatisfatória do PNVITA e falta de orientação sobre a periodicidade da administração da suplementação. ${ }^{12}$

É importante, para o sucesso do Programa, que as puérperas e as mães e/ou responsáveis por crianças tenham informação sobre a periodicidade da administração da vitamina A, sobre a faixa etária de administração e que sejam capazes de identificar a data de retorno às unidades de saúde. ${ }^{4,9} \mathrm{~A}$ adesão da população às ações de saúde, tornando-as mais eficazes, precisa de uma maior interação entre os especialistas (acadêmicos e/ou técnicos) e a comunidade, e necessitam ser condizentes com o cotidiano dessa população. ${ }^{24}$

\section{CONCLUSÃO}

O desconhecimento e a invisibilidade das beneficiárias sobre o PNVITA e seus componentes indicam a necessidade de sensibilização da comunidade para participar ativamente do Programa, além da distribuição de cápsulas de vitamina A. Despertar a motivação, entendida aqui no sentido do interesse e da curiosidade, e prender a atenção é primordial para mudar comportamentos e incentivar a participação. Nesse sentido, as atividades educativas devem ser desenvolvidas de modo a permitir que a 
população-alvo seja sujeito da aprendizagem, e o profissional de saúde, facilitador desse processo.

As ações educativas voltadas ao conhecimento sobre a vitamina $A$, sua deficiência e o Programa, partindo de conhecimento popular, hábitos, condutas pessoais e coletivas, podem contribuir para que a comunidade se sinta corresponsável em relação à prevenção e controle da DVA. Entende-se que as atividades educativas são ferramentas indispensáveis para a participação ativa da população e o fortalecimento do Programa na realidade investigada. Essas ações devem percorrer a assistência prénatal, o puerpério e a puericultura, de forma que toda população-alvo do Programa seja contemplada. Sugere-se, ainda, a necessidade de padronizar o registro da vitamina A na Caderneta de Saúde da Criança, para beneficiários e profissionais poderem identificar a periodicidade da suplementação e o retorno, além de reforçar a divulgação do Programa.

Na perspectiva do profissional, desprende-se a necessidade de mudanças nas práticas mecanicistas distantes da perspectiva do autocuidado, da promoção de saúde e do trabalho em equipe multiprofissional. É importante que o profissional de saúde desempenhe seu papel social, contribuindo, além dos procedimentos clínicos e assistenciais, na qualidade de vida das pessoas. A educação permanente constitui uma estratégia essencial no aperfeiçoamento dos conhecimentos dos profissionais de saúde e com mudanças em suas práticas. O envolvimento dos profissionais de saúde é fundamental para assegurar o cumprimento das ações preconizadas pelo Programa e favorecer a participação ativa dos beneficiários.

Ponderando que a participação ativa da população-alvo no Programa é importante para sua operacionalização, novas pesquisas devem ser realizadas sobre o assunto em outros municípios. Sugere-se, também, a necessidade de uma avaliação do Programa de âmbito nacional, uma vez que seu funcionamento adequado é imprescindível para o controle e a prevenção da DVA.

\section{AGRADECIMENTOS}

Os autores agradecem à Secretaria Municipal de Saúde de Campina Grande pela permissão para a realização da pesquisa e à Fundação de Apoio à Pesquisa do Estado da 
Paraíba (Fapesq) pelo financiamento concedido.

\section{CONTRIBUIÇÃO DOS AUTORES}

Dixis Figueroa Pedraza participou da concepção do estudo, da redação e da revisão crítica do manuscrito; e da interpretação dos dados. Juliane Berenguer de Souza Peixoto participou da redação e da revisão crítica do manuscrito; e da análise e da interpretação dos dados. Maria Gorete Lucena de Vasconcelos participou da revisão crítica do manuscrito; e da interpretação dos dados. Virgínia Rossana de Sousa Brito participou da revisão crítica do manuscrito; e da análise e da interpretação dos dados. Adriana de Azevedo Paiva participou da concepção do estudo e da revisão crítica do manuscrito; e da interpretação dos dados. Todos os autores aprovaram a versão final do manuscrito e declaram ser responsáveis por todos os aspectos do trabalho, garantindo sua exatidão e sua integridade.

\section{REFERÊNCIAS}

1. Martins MC, Oliveira YP, Coitinho DC, Santos LMP. Panorama das ações de controle da deficiência de vitamina A no Brasil. Rev Nutr. 2007 ; 20(1):5-18.

2. Queiroz D, Paiva AA, Figueroa Pedraza D, Cunha MAL, Esteves GH, Luna JG et al. Deficiência de vitamina $A$ e fatores associados em crianças de áreas urbanas. Rev Saúde Públ. $2013 ;$ 47(2):248-56.

3. Ramalho RA, Padilha P, Saunders C. Análise crítica de estudos brasileiros sobre deficiência de vitamina A no grupo materno-infantil. Rev Paul Pediatr. 2008; 26(4):392-9.

4. Brasil. Manual de Condutas Gerais do Programa Nacional de Suplementação de Vitamina A. Brasília: Ministério da Saúde; 2013.

5. Brasil. Pesquisa Nacional de Demografia e Saúde da Criança e da Mulher - PNDS 2006: dimensões do processo reprodutivo e da saúde da criança. Brasília: Ministério da Saúde; 2009.

6. Silva LLS, Peixoto MRG, Hadler MCCM, Silva AS, Cobayashi F, Cardoso MA. Estado nutricional de vitamina $A$ e fatores associados em lactentes atendidos em Unidades Básicas de Saúde de Goiânia, Goiás, Brasil. Rev Bras Epidemiol. 2015 ; 18(2):490502.

7. Figueroa Pedraza D, Queiroz D, Paiva AA, Cunha MAL, Lima ZN. Seguridad alimentaria, crecimiento y niveles de vitamina $A$, hemoglobina y zinc en niños preescolares del nordeste de Brasil. Ciênc Saúde Colet. 2014 ; 19(2):641-50.

8. Lima DB, Damiani LP, Fujimori E. Deficiência de vitamina A em crianças brasileiras e 
variáveis associadas. Rev Paul Pediatr. 2018 ; 36(2):176-85.

9. Brasil. Carência de Micronutrientes. Brasília: Ministério da Saúde; 2007. (Série A. Normas e Manuais Técnicos).

10. Martins MC, Santos LMP, Santos SMC, Araújo MPN, Lima AMP, Santana LAA. Avaliação de políticas de segurança alimentar e combate à fome no período 19952002. 3 - O Programa Nacional de Controle de Deficiência de Vitamina A. Cad Saúde Públ. 2007; 23(9):2081-93.

11. Almeida ER, Carvalho AT, Nilson EAF, Coutinho JG, Ubarana JA. Avaliação participativa do Programa Nacional de Suplementação de Vitamina A em um município da Região Nordeste do Brasil. Cad Saúde Públ. 2010; 26(5):949-60.

12. Paiva AA, Cagliari MPP, Queiroz D, Souto RA, Brito BRS, França ISX. Programa Nacional de Suplementação da Vitamina A no Estado da Paraíba: uma análise a partir do relato de profissionais da Equipe de Saúde da Família. Epidemiol Serv Saúde. 2011; 20(3):378-83.

13. Instituto Brasileiro de Geografia e Estatística. Censo Demográfico 2010. [Internet]. Rio de Janeiro: IBGE [citado 2014 out 7]. Disponível em: <http://www.ibge.gov.br/cidadesat/topwindow.htm?1>.

14. Brasil. Ministério da Saúde. Cadastro Nacional de Estabelecimentos de Saúde. Estabelecimento de Saúde do Município: Campina Grande [Internet]. Brasília: Ministério da Saúde [citado 2014 out 7]. Disponível em: http://cnes2.datasus.gov.br/Lista_Es_Municipio.asp?VEstado=25\&VCodMunicipio= 250400 \&NomeEstado=PARAIBA.

15. Fontanella BIB, Ricas J, Turato ER. Amostragem por saturação em pesquisas qualitativas em saúde: contribuições teóricas. Cad Saúde Públ. 2008; 24(1):17-27.

16. Boni V, Quaresma SJ. Aprendendo a entrevistar: como fazer entrevistas em ciências sociais. Rev Eletrôn dos Pós-graduandos Sociol Polít UFSC. 2005; 2(1): 68-80.

17. Bardin L. Análise de Conteúdo. Lisboa: Edições 70; 2009.

18. Rios ERG, Franchi KMB, Silva RM, Amorim RF, Costa NC. Senso comum, ciência e filosofia - elo dos saberes necessários à promoção da saúde. Ciênc Saúde Colet. $2007 ; 12(2): 501-9$.

19. Junges JR, Barbiani R, Soares NA, Fernandes RBP, Lima MS. Saberes populares e cientificismo na estratégia saúde da família: complementares ou excludentes? Ciênc Saúde Colet. 2011; 16(11):4327-35.

20. Jahn AC, Guzzo PC, Costa MC, Silva EB, Guth EJ, Lima SBS. Educação popular em saúde: metodologia potencializadora das ações do enfermeiro. Rev Enferm UFSM. 2012; 2(3);547-52.

21. Rodrigues LPF, Roncada MJ. A educação nutricional nos programas oficiais de prevenção da deficiência da vitamina A no Brasil. Rev Nutr. 2010; 23(2):297-305.

22. Fontes BA, Lima R, Lima JA. Promoção de saúde e participação social: o modelo de atenção básica do sistema de saúde brasileiro. Cienc Sociais Unisinos. 2010; 46(1):65-79. 
23. Brito VRS, Vasconcelos MGL, Diniz AS, França ISX, Figueroa Pedraza D, Peixoto JBS, PAIVA AA. Percepção de profissionais da saúde sobre o Programa Nacional de Suplementação de Vitamina A. Rev Bras Promoç Saúde. 2016;29(1):93-9.

24. Cavalcanti CCTJ. Aproximando a lógica sanitária e a lógica do senso comum: uma experiência e-learning em prevenção à dengue na comunidade da cidade universitária da USP. [Dissertação]. São Paulo: Faculdade de Saúde Pública da USP; 2010.

Submissão: agosto de 2016.

Aprovação: dezembro de 2018. 